\title{
Wertschätzung für den alten Menschen
}

\author{
Preisträgerin des Alois-Alzheimerpreises 2009 ist die Gerontologin Naomi Feil
}

Herausragende wissenschaftliche, publizistische oder individuelle Leistungen werden mit dem Alois-Alzheimerpreis gewürdigt. Die Preisträgerin 2009 ist Naomi Feil. Die feierliche Ehrung mit einer Laudatio von Maria Hoppe, Gründungsmitglied des Österreichschen Instituts für Validation, fand Ende November im Wiener Rathaus statt.

Validation ist eine Kommunikationsmethode, um mit verwirrten, sehr alten Menschen umzugehen. Sie wurde zwischen 1963 und 1982 von Naomi Feil entwickelt und 1989 in Europa eingeführt. Seit den ersten Workshops in Wien im März 1989, in Apeldoorn im September 1989 und der Publikation von Feils erstem Buch Validation: The Feil Method (1989: Übersetzung ins Deutsche unter dem Titel Validation, ein Weg zum Verständnis verwirrter alter Menschen) wurde diese Methode oft kritisiert. Kritik regt dazu an, neue Ideen zu entwickeln, alte zu konkretisieren oder anzupassen, sie zu vertiefen.

Die erste und wahrscheinlich bedeutsamste Veränderung war der Wechsel von der Bezeichnung „Validationstherapie“ zu „Validationsmethode“. Während in den USA das Wort „Therapie“ sehr häufig im Zusammenhang mit Behandlungen und Methoden verwendet wird, geht man in Europa mit dem Gebrauch dieses Wortes sorgfältiger um (in gewissen Fällen ist der Gebrauch rechtlich eingeschränkt) und impliziert damit immer auch eine medizinische Heilung. Diese kritische Hinterfragung führte dazu, die Rolle der Validation sowohl in der psychogeriatrischen Pflege stärker zu berücksichtigen als auch ihre Ziele und Resultate besser zu definieren. Validation wird nun als ein Prozess gesehen, der durch starkes Vertrauen wächst.

\section{Geborgenheit und Vertrauen}

Validationsexperten arbeiten immer „state of the art". Ein Validationsanwender beginnt damit, ein geborgenes Umfeld zu schaffen, in dem die Beziehung zum Klienten stattfinden soll. Er erzeugt Vertrauen, ohne zu urteilen und erforscht die-

\footnotetext{
1 Mag. Maria Gattringer

Ausbildungszentrum des Wiener Roten Kreuz
}

persönliche Realität des Anderen. Dies führt zu einem empathischen Austausch von Gefühlen, wobei der Klient sich dazu ermutigt fühlt, sich sowohl verbal als auch nonverbal auszudrücken. Die Hoffnung besteht darin, dass der Klient wieder an Selbstwertgefühl gewinnt, sich durch die Äußerung bisher unterdrückter Gefühle erleichtert fühlt und in seinem Prozess der Aufarbeitung unerledigter Aufgaben (aus der Vergangenheit) begleitet wird.

\section{Das „Leben“ der Validation}

Die Validation kann mit der menschlichen Entwicklung verglichen werden; sie wurde 1989 geboren und ist seitdem als Methode gereift, tiefgründiger und komplexer geworden. Sie wurde durch Kritik herausgefordert, worauf sie mit Selbstprüfung reagierte und sich dadurch in vielen Fällen zum Besseren

\section{„Validation“ fördert eindeutig} das Wohlbefinden von demenzkranken Menschen, aber auch das Wohlbefinden ihrer sie betreuenden Familien.

veränderte. Veränderung fand langsam und überlegt statt. Im Veränderungsprozess waren ganz unterschiedliche Personen aus verschiedenen Ländern involviert, alle sachkundig und im Bereich der psychogeriatrischen Pflege hoch qualifiziert - und sehr engagiert für das Wohl desorientierter Alter. Schließlich sind sie es, für die sich Validationsexperten engagieren.

Dennoch, Validation muss gelehrt und gelernt werden. Dazu dienen Basisschulungen, um sich mit dem Gedankengut von Naomi Feil auseinander zu setzen. Sie ermöglichen, bereits einzelne Validationstechniken in der Praxis anzuwenden. Zum Experten wird man durch eine Ausbildung zum Validationsanwender, die Kommunikationsprobleme validierend lösen und so zum Wohlbefinden ihrer Klienten beitragen können. Die nächste Stufe der Ausbildung ist der Gruppenleiter. Gruppenleiter ermöglichen demenzkranken Menschen einmal in der Woche „auszugehen“. Indem sie die Validationsgruppe besuchen, werden sie wieder zu den Persönlichkeiten, die sie vor der
Erkrankung waren. Nach der Gruppe kehren sie fröhlicher und kommunikativer in den Alltag zurück. Ein Gruppenleiter fördert damit die Lebensqualität von demenzkranken Menschen enorm.

\section{Standards und Zertifzierung}

Naomi Feil hat aber auch einen Lehrplan für die Lehrerausbildung mit vorgegebenen Lehr- und Lernzielen oder Standards entwickelt. „Zertifizierter Validationslehrer" darf sich nur nennen, wer diesen Standards entspricht. Das heißt, die Lehrerausbildung baut auf der Anwenderund Gruppenleiterausbildung auf. Sie ist eine sehr anspruchsvolle Weiterbildung welche vorerst zum „Presenter“ führt. Zum Lehrer wird man erst nach Abschluss eines Co-Trainings, in dem der zukünftige Lehrer in Zusammenarbeit mit einem erfahrenen Lehrer einen Lehrgang durchführen muss. Diese strengen Maßnahmen gewährleisten, dass überall, wo Validation nach Naomi Feil angeboten wird, auch „Validation nach Naomi Feil“ drinnen ist. Unklarheiten dazu beantworten die einzelnen Autorisierten Validationsorganisationen Österreichs (EVA). (www.validation-eva.com)

Seit 45 Jahren arbeitet Naomi Feil erfolgreich mit der Validationsmethode. Ihre wissenschaftliche Leistung oder die „Validation" fördert eindeutig das Wohlbefinden von demenzkranken Menschen, aber auch das Wohlbefinden ihrer sie betreuenden Familien. Für alle Experten auf diesem Gebiet ist sie der Baustein für eine bessere Kommunikation im Alltag. Der Alois-Alzheimer-Preis wurde damit 2009 einer großen Persönlichkeit und Wissenschaftlerin verliehen. Sie vereint mit ihrer Leistung alle Disziplinen, die demenzkranke Menschen therapieren, betreuen und umsorgen.

Informationen zu Validation: ABZ Ausbildungszentrum des Wiener Roten Kreuzes GmbH Tel.: 01/795 80-6300; Fax: 01/795 80-59 6502 E-Mail: abz-kundenzentrum@w.roteskreuz.at; Internet: www.w.roteskreuz.at/abz

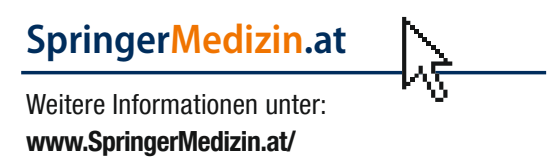

